\title{
Hardware and software evolution of an adaptive ability measurement system
}

\author{
LOUIS J. DEWITT and DAVID J. WEISS \\ University of Minnesota, Minneapolis, Minnesota 55455
}

\begin{abstract}
A software system for the administration of six different strategies of adaptive ability tests in a variety of formats was developed both on a large-scale time-sharing system and on a real-time minicomputer system. Factors influencing both hardware selection and software design are discussed.
\end{abstract}

For over half a century, the prevailing method of ability measurement has been the administration of multiple-choice paper and pencil tests to groups of individuals. The deficiencies of this form of testing, along with the growing availability of sophisticated computer equipment, have led to the development of a number of new testing strategies. These new strategies combine the presentation of a test item on a peripheral device such as a CRT with the ability of the computer to evaluate the subject's response and to select the next item to be presented, using some set of predetermined rules. Such testing systems are commonly called computer-assisted or adaptive testing systems. Adaptive tests have a potential for yielding more reliable, precise, and valid test scores than conventional paper and pencil tests as well as doing so with fewer items. There are a number of other potential advantages. Among these: the elimination of time limits may reduce artifactual speed components in test scores; the presentation of test items of a difficulty adapted to the subject's ability level may reduce test anxiety or boredom; the use of knowledge of results (feedback) after each item may increase motivation; and the use of item-response latency data may lead to additional diagnostic or predictive information. The primary benefits, however, are better and shorter tests.

At The University of Minnesota, we have been doing research on computer-assisted adaptive testing since 1969. The purpose of this paper is to discuss some of our experiences and thoughts concerning both c $\lrcorner$ mputer hardware and software as they relate to our research goals. To this end, it is possible to look at three distinct phases in our research which can be labeled: (1) initial research, (2) development of a software system, and (3) moving to a minicomputer system.

The research reported in this paper was supported by Contract No. N00014-67-A-0113-0029, NR No. 150-343, with the Personnel and Training Research Programs, Psychological Sciences Division, Office of Naval Research.

\section{INITIAL RESEARCH}

Before any research was begun, a decision was made to use multiple-choice vocabulary items in the initial phases. This permitted us to use character mode CRTs which were available on several systems without the need for acquiring and interfacing specialized computer equipment. Two goals were then set for our initial research: (1) construction of an item pool normed on the subject pool with which we would be working (the proverbial introductory psychology class) and (2) establishment of equivalency between conventional paper and pencil tests and similar computer-presented tests. A single program was developed and implemented on an IBM 1130 with a 1500 CRT display system for collecting data. Data was collected for several hundred subjects and stored on punched cards. This system was used for several experiments during the course of the year.

The subsequent unavailability of this system led us to adapt our program to a CDC 3200 . This was a powerful real-time system with foreground and background partitions. It served primarily as a remote job entry station to the University's CDC 6600 . Little software was available for driving CRTs, and it was necessary for us to expend considerable effort toward developing pseudo-time-sharing software to drive several CRTs simultaneously.

This system proved to be fast; it was possible while testing our software to cycle through three items per second on a CRT. Accurate latencies on subject responses were also collected. There were, however, several deficiencies. Due to the system's function as an RIE station, our system development and maintenance could only be scheduled between midnight and 8:00 a.m. When the system was pushed hard by its RJE functions and our testing, it had an annoying tendency to "lose interrupts," after which it would grind to a halt. Recovery required only a few minutes, but as we had no recovery software, we could lose all subjects who were testing at the time. The system was in heavy demand and usually only $2 \mathrm{~h} / \mathrm{day}$ 
could be scheduled. If hardware difficulties necessitated a late start and our subjects were tesing past our scheduled time, they would be unable to complete a test, as the program would be terminated by the operators. A final deficiency was that no hard-copy output device other than a printer was available; i.e., there were no punched cards or magnetic tapes. With some effort, it was possible to submit one of our own disk files as a job for export to the CDC 6600, where the data could be stored on tape for later analysis. This, however, was never a very satisfactory method. In spite of these difficulties, we administered tests to several hundred subjects in a year's time and saved their data on tape.

By the start of the next school year, a State educational time-sharing network had become operational. We leased three CRTs, acoustic couplers, and 30-character/sec ports and continued testing for item norming using simple programs.

\section{DEVELOPMENT OF A SOFTWARE SYSTEM}

As our item pool grew and our research goals developed, we began defining the requirements of a softuare system for adaptive testing. Among these requirements were operational simplicity, software tlexibility, recoverability, and economy of both central memory usage and disk file operations and storage. Operational simplicity meant that proctors with little training could easily log subjects on and start them testing. that the system would be self-instructional, ensuring that all subjects could operate the equipment satisfactorily, and that the occasional unreadable screen could easily be displayed a second time. Software flexibility required that a subject's testing conditions could be chosen by the proctor at the time the subject was logged on. A testing condition could involve one or two tests, each of which could be one of six available testing strategies, each using one of several unique item pools, and with each test providing no feedback, feedback after each item, or feedback upon completion of testing. Flexibility also required that, as they were developed, new strategies of testing could be easily integrated into the system along with their unique item pools. Recoverability was dictated by the inevitability of system crashes. With recovery routines, it would be possible to resume testing of a subject at the point of interruption after a system crash. Economy of central memory usage and disk operations was necessary to keep system response time as short as possible. Economy of disk file storage was necessary as daily storage charges were based on file size. The design and implementation of this system were described in a technical report (DeWitt \& Weiss, Note 1).

This system was used for over 2 years, collecting data on over 2,000 subjects. As our testing progressed, however, so did our dissatisfaction with the system. This dissatisfaction resulted mainly from problems caused by the characteristics of our subject pool. The size of the pool varied from quarter to quarter, with fall quarter generally yielding the largest number of subjects. Subjects were awarded points toward their final grades. They were required to be available for $1 \mathrm{~h}$ of experiment time and were to be awarded points for presenting themselves at the required place and time. We generally scheduled three or four subjects per hour. Thus, when these subjects came for testing and the computer system happened to crash, we had no alternative but to award thern thei points and let them go. Even if the subjects had no class the following hour and could wait until the system was available, they might continue testing into the next hour. which would raise more conflicts. This was a continuing problem, but during 1 month, while a new operating system was being debugged, we probably lost a quarter of our potential subjects for these reasons.

A second major deficiency of this system was system response time - the delay between pressing the return key and receiving output on the screen. As the number of ports on the system grew, it was not uncommon to have over 100 active users on the system during peak periods. This large number of users caused a drastic increase in response time. System response times were usually $15-20 \mathrm{sec}$, with $40-45 \mathrm{sec}$ not uncommon. Probably half of our subjects' time was spent waiting for the system to respond. If our experimental design involved feedback, which required two responses per item, we were limited to short tests to avoid using more than $1 \mathrm{~h} /$ subject, or having the subjects leave before completing testing. For the CAI student or programmer, there was sufficient motivation to persevere or return later, but this was rarely the case for our subjects. It should also be obvious that latency measurement under these conditions would be almost meaningless. The attrition of our available subjects in addition to the time constraints led us to investigate the feasibility of a minicomputer system for our testing.

\section{DEVELOPING A MINICOMPUTER SYSTEM}

In January of 1974, we set up a schedule which allowed us 3 months to investigate the available minicomputer systems and place an order, 3 months for delivery, during which time we could begin designing our software, and 3 months for system development and debugging, which would enable us to begin testing early in October. The inevitable delays kept us from starting until the middle of November, $1 \frac{1 / 2}{2}$ months behind schedule.

The system we selected is a Hewlett-Packard 9600E real time data acquisition system based on their $2100 \mathrm{~S}$ 
minicomputer, with 32,768 16-bit words of memory. Our peripheral equipment includes a Teletype, a high-speed paper-tape reader, a disk with one fixed and one removable platter, each capable of storing 2.47 million characters, four 960-character/sec CRTs, one 240-character/sec CRT, one 30character/sec CRT and thermal printer, and synchronous communications equipment.

Our software is based on Hewlett-Packard's RTE-I operating system and includes an interactive text editor, a file management system, a Fortran IV compiler, assembler, loader, and a software package which simulates a CDC U200 terminal, giving us access to the University's CDC CYBER 74 as an RJE station. The operating system allows for foreground and background partitions with program swapping in foreground. Our present configuration allocates approximately one-third of the memory to the operating system, one-third to foreground programs, and one-third to background. Our entire testing system. consisting of six core-resident and five disk-resident programs, operates entirely in foreground memory.

Several features of the system have proven important to us. The fact that we have two disk platters plus disk-to-disk copy routines enables us to make backup copies of our disks. In the event of some catastophic disk failure, we would have little trouble reconstructing the system. The ability to communicate with the CDC CYBER 74 eliminates the need for a printer or tape drive. Using the interactive text editor, disk files can easily be prepared and then sent to the CYBER 74 for listing on an RJE station just down the hall, or to permanent storage on magnetic tape. Conversely, it is possible to receive tape or card files from the CYBER 74 and store them directly on disk. The fact that the testing system operates in foreground core leaves background core free for text entry and editing, program compilation, loading and execution, data preparation and transmission to the CYBER 74. All of these functions are carried out on our 240-character/sec CRT, which can serve as a console when needed.

Several members of our staff are interested in computer simulation studies and have developed a simulation software system which can generate responses for tens of thousands of hypothetical subjects, then analyze the data and print out results. This simulation system can be set up to run unattended for hours at night or over a weekend. These studies proved unwieldy on a time-sharing system and expensive on a batch-processing system, but are essentially free on our minicomputer.

The elimination of expensive peripheral equipment enabled us to keep costs low. The system, which is being purchased on an educational time-pay plan, costs less per year than four ports on our previous time-sharing system would cost. The two slower CRTs are owned by our department, so a programmer console is available at no extra cost. We have no disk storage costs beyond the one-time purchase cost of extra disk packs.

The reliability and stability of our system has been highly satisfactory. During our first 2 months of testing, we were plagued with system crashes approximately once per week. Restarting the system took only a minute or two, but we had no recovery routines, so the data for all subjects currently testing was lost. A disk hardware failure eventually brought us to a complete halt, and the repair of that problem eliminated our crashes. During the next 3 months, we experienced no crashes, but occasionally all our CRTs would stop responding, in a deadlock, each waiting for the other to be processed. This was traced to a software error on our part, which was not corrected at the time because of its infrequency.

Several features of the system were changed prior to beginning testing this fall and so far we have tested approximately 400 subjects with no abnormal terminations. We have experienced only the one hardware failure in 15 months of operation. Occasional CRT failures are to be expected but usually do not involve the loss of subjects. Response time, as our system is currently configured, averages $.25 \mathrm{sec}$, eliminating system response time as a consideration in experimental designs and yielding accurate response latency measurement.

\section{SOME FEATURES OF OUR CURRENT TESTING SYSTEM}

Our first year's experience on a minicomputer system led us to evaluate some of its strengths and weaknesses and to modify our software system accordingly. The overall reliability of the system has led to a decision not to incorporate recovery routines into the testing system. The intensive disk usage of the testing system and the sensitivity of the system to disk errors, whether software or hardware induced, led us to reevaluate our method of accessing data on disk. The reevaluation, along with the realization of some of the inefficiencies of the vendor-supplied file management software, led us to rewrite the disk accessing routines with emphasis on eliminating all possible errors. A specialized file management program was written to permit easy modifications and additions to our item pool, which contains approximately 1,200 items.

The fast system response time enabled us to collect more data per subject hour. Accordingly, the testing system was modified to permit the administration of three tests to each subject. In addition, each test can be followed by a series of questions designed to get at the subject's reactions to the test he/she has 
completed. Instructions can also be presented for the following test. Our experimental designs can become much more comprehensive because larger numbers of subjects can be tested.

As a result of the increased system flexibility, it became advantageous to add a test construction program to the system. This program enables the researcher at a CRT to interactively create an experimental testing condition. Up to 30 variables defining the test condition are obtained from the researcher and checked for errors before being posted on disk. When a subject is available for testing, the proctor need only enter a two-character key to call up the predefined testing condition. This test construction program also maintains statistics on the number of uses of, dates of creation of, and last access of each testing condition, enabling the researcher to have the current status of the quarter's research displayed.

A brief description of our research for this fall may help to illustrate the flexibility of the current system. We had previously defined, and stored on disk, 14 experimental conditions to be administered to $700-800$ subjects. When a subject presents himself for testing, the proctor enters a special code instructing the system to select one of the 14 conditions. The subject then receives a series of instructional screens to acquaint him with the equipment and to collect biographical data such as name, age, and ID number. A file is then created, keyed to the subject's unique ID number. If, at any point during the instructions or test, the subject fails to enter a valid response after three consecutive tries, a bell is sounded on the CRT and the subject is instructed to summon the proctor. who then gives him assistance.

Upon completing the instructional sequence, administration of the first test is begun. This test is one of seven strategies and is administered with or without inter-item feedback. Upon completion of this first test, which is usually about 50 multiple-choice vocabulary items, the subject receives 18 questions attempting to ascertain his feelings of anxiety, motivation, and other reactions to the experimental conditions. If the subject received feedback, an additional eight questions are presented to ascertain his reactions to receiving feedback.
Following these post-test questions, instructions are presented for the second of the three tests. The second test consists of 20 free-response vocabulary items in which the subject is required to enter the word most similar to the stimulus word. Upon completion of this test, the subject receives instructions for the third test, which is a 32-item multiple-choice test with no feedback.

Upon completion of the third test, the subject may elect to receive a series of screens explaining the goals of the experiment. The screens presented are adapted to the subject's experimental condition. The subject is then free to leave. Upon completion of an experiment, the subject's data files are processed and converted into a card image format which is transmitted to the CYBER 74 for storage on tape and later analysis. For debugging and illustrative purposes, a number of programs which display a subject's test record graphically are available on the test system.

\section{FUTURE PLANS}

Most of our research to date has centered around the strategies used to select items that are displayed and scored in a method similar to paper and pencil tests, and are directed at ability measurement. Research remains to be done on performance measurement and norm-referenced vs. criterionreferenced testing. Within this framework, the potential for different item types is to be explored. Multistage items using within-item branching and/or differential response scoring are to be investigated. Finally, different methods of item presentation are to be developed. We are currently investigating the feasibility of interfacing Plato terminals to our system. These terminals, with their graphics and microfiche projection capabilities, should permit us to develop new forms of items for testing as well as new forms of tests.

\section{REFERENCE NOTE}

1. DeWitt, L. J., \& Weiss, D. J. A computer software system for adaptive ability measurement. Research Report 74-1, Psychometric Methods Program, Department of Psychology. University of Minnesota, 1974. (AD 773961) 